\title{
A case of virilization and bilateral Krukenberg tumours in late pregnancy.
}

\author{
Jing-Min Wang, Fang-Xin Shi* \\ Department of Obstetrics and Gynaecology, the First Affiliated Hospital of Dalian Medical University, Dalian 116011, \\ Liaoning Province, PR China
}

\begin{abstract}
A Krukenberg tumour (KT) is an ovarian cancer mainly derived from metastasis of gastrointestinal tract cancers. While the incidence of $\mathrm{KT}$ is low, the prognosis is poor. We report a case of a gravida 1 , para 0 woman at 36 weeks of gestation who had bilateral, large ovarian masses, virilization, and hirsutism. She had an uneventful spontaneous vaginal delivery and died 12 months postpartum, despite having received a full course of chemotherapy. Obstetricians should pay close attention to any gastrointestinal symptoms that occur during pregnancy, especially in patients presenting with bilateral ovarian tumours, as early diagnosis or detection of KT and primary lesions could significantly improve the prognosis.
\end{abstract}

Keywords: Krukenberg tumour, Stomach neoplasms, Maternal death, Pregnancy complication.

Accepted on December 04, 2017

\section{Introduction}

Krukenberg tumour (KT) is an ovarian adenocarcinoma that metastasizes from a primary malignancy of the gastrointestinal tract, with $76 \%$ originating in the stomach. $\mathrm{KT}$ is bilateral in $80 \%$ of cases [1]. KT was first reported in 1896 by the German pathologist Friedrich Ernst Krukenberg [2]. He described these tumours as bilateral, usually of medium size, and kidneyshaped. The tumours are generally solitary rather than multiple, and are often associated with ascites. KT diagnosis is confirmed microscopically by the presence of a typical signet ring cell. These tumours are therefore also referred to as signet ring cell tumours (signet ring cell adenomucinous carcinomas) [3]. KTs are highly malignant and the prognosis is poor, with a median survival of 14 months [4].

The overall incidence of gastric cancer in women of reproductive age is low ( 0.4 to $0.5 \%)$ [5]. KT accounts for 1 to $2 \%$ of all ovarian neoplasms [6]. A small number of patients present with endocrine manifestations including virilization, hirsutism, breast soreness and swelling, postmenopausal vaginal bleeding, and endometrial hyperplasia [7].

We present the case of a woman with bilateral ovarian masses, virilization, and hirsutism, who underwent an uneventful, spontaneous vaginal delivery at 36 weeks. Despite treatment with chemotherapy, this patient died 12 months postpartum.

\section{Case Presentation}

A 30-year-old woman (gravida 1, para 0) was admitted to the obstetrics unit at 36 weeks of gestation. The patient complained of recurrent abdominal pain, vomiting, and melena for three months and hematemesis for one day. By 12 weeks of gestation, she had developed a husky voice and facial acne, both of which worsened before admission. This study was conducted in accordance with the declaration of Helsinki. This study was conducted with approval from the Ethics Committee of the First Affiliated Hospital of Dalian Medical University. Written informed consent was obtained from participant.

By 24 weeks of gestation, her skin colour had darkened, and she developed a facial beard as well as back and limb pain. The vomiting worsened as the disease progressed, and she could only consume liquids, inevitably resulting in weight loss. Despite these symptoms, the patient had not previously sought medical attention.

Transabdominal colour Doppler examination at 16 weeks of gestation showed a left abdominal mass measuring $3.7 \times 3.0 \times$ $3.0 \mathrm{~cm}$. At 31 weeks of gestation, repeat examination showed bilateral, solid, kidney-shaped ovarian masses. Both ovaries were highly vascularized, with the left measuring $8.1 \times 6.2 \times$ $4.8 \mathrm{~cm}$ and the right measuring $8.5 \times 6.9 \times 4.7 \mathrm{~cm}$. At 33 weeks of gestation, the left ovary had grown to $8.8 \times 6.9 \times 5.2$ $\mathrm{cm}$ and the right ovary had grown to $9.5 \times 7.2 \times 6.4 \mathrm{~cm}$. Unfortunately, during routine prenatal care at another hospital, her symptoms had been attributed to the underlying pregnancy.

The patient reported a 10-year history of gastroesophageal reflux and eructation prior to pregnancy, which had been examined by upper gastrointestinal endoscopy four years prior to her current admission to the obstetrics unit. These symptoms were diagnosed as being associated with gastritis, and intermittent acid suppression therapy was advised. The patient denied use of alcohol, cigarettes, or illicit drugs, and had no family history of malignant disease. 
At physical examination upon admission, the patient appeared mildly anaemic and her height and weight were $176 \mathrm{~cm}$ and 74 $\mathrm{kg}$, respectively. She had a husky voice, a receding hairline, and widespread, pigmented acne was observed on her face, neck, chest, and back. She also had a black beard on her jaws and cheeks. Neck abnormalities and swollen lymph nodes were not observed. Auscultation of the chest did not reveal any obvious abnormality of the heart or lungs. Her abdominal circumference was $96 \mathrm{~cm}$ and uterine height was $31 \mathrm{~cm}$, with a foetal heart rate of 140 beats per min. The patient had obvious, bilateral pitting edema on her lower limbs.

Laboratory tests revealed the following: haemoglobin, $8.5 \mathrm{~g} / \mathrm{dl}$; circulating testosterone, $>15 \mathrm{ng} / \mathrm{ml}$ (normal range: $0.06-0.82$ $\mathrm{ng} / \mathrm{ml}$ ); adrenocorticotropic hormone (ACTH), $16.85 \mathrm{pg} / \mathrm{ml}$ (normal range: 7.2-63.3 pg/ml); and cortisol, $829.97 \mathrm{nmol} / \mathrm{L}$ (normal range: 118.6-518 nmol/L). CA 125, CA 199, and carcinoembryonic antigen levels were $117.5 \mathrm{U} / \mathrm{ml}, 53.69 \mathrm{U} / \mathrm{ml}$, and $4.75 \mathrm{ng} / \mathrm{ml}$, respectively.

At 37 weeks of gestation, sudden, premature rupture of the membranes led to the spontaneous delivery of a healthy male infant weighing 3,250 g, with Apgar scores of 10 at both 1 and 5 minutes. The total duration of labour was $7 \mathrm{~h}$ and $15 \mathrm{~min}$, with no complications.

Computed tomography (CT) was performed after delivery to investigate the cause of the signs and symptoms, including the virilization and bilateral abdominal masses. CT revealed diffuse thickening of a region of the stomach wall (approximately $2.62 \mathrm{~cm}$ ), with associated bilateral, irregular masses that were cystic and solid in nature. A left lateral cyst measured $8.85 \times 8.85 \mathrm{~cm}$ and a right lateral cyst measured 9.84 $\times 9.84 \mathrm{~cm}$. CT also showed signs of ascites (Figure 1).

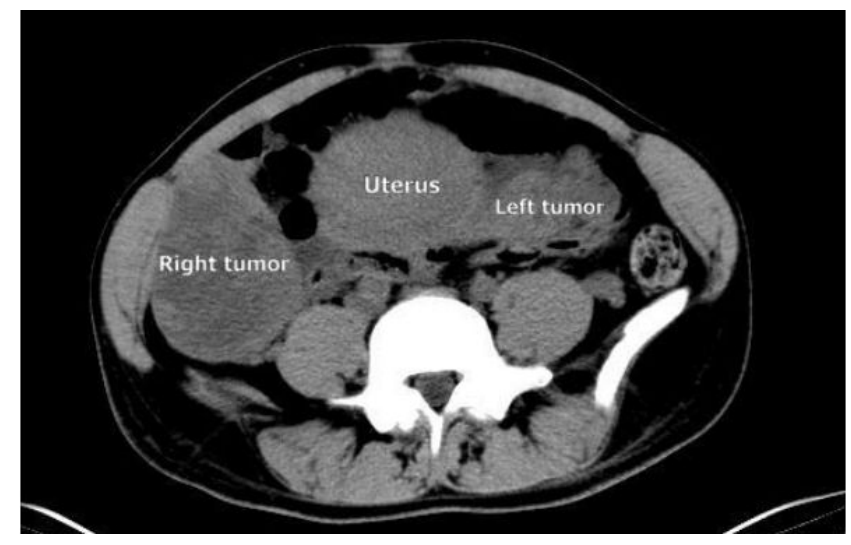

Figure 1. Abdominal CT scan showing bilateral adnexa masses.

Gastrointestinal endoscopy performed after the delivery revealed a tubulovillous adenoma in the gastric antrum, as well as a well-defined, deep, medial ulcer in the lesion itself. A biopsy was performed, and microscopic examination revealed diffuse adenocarcinoma with signet ring cells.

To ensure a valid and accurate diagnosis, transabdominal ultrasound-guided ovarian puncture and biopsy were performed for pathological analysis. The results showed numerous cancerous cell nests in the ovarian stroma, as well as the characteristic morphology of signet ring cells (mucus-filled heterocysts with nuclei pushed towards the periphery of the cells) (Figure 2). Oncological therapy was recommended, as surgical resection of the tumours could be fatal, considering the patient's postpartum weakness and rapid deterioration. In the postpartum period, the patient's testosterone level decreased to $0.143 \mathrm{ng} / \mathrm{ml}$; the acne, which was initially localized to the face and upper posterior trunk, spread over her entire body, and the facial beard continued to grow, reaching a length of $2 \mathrm{~cm}$.

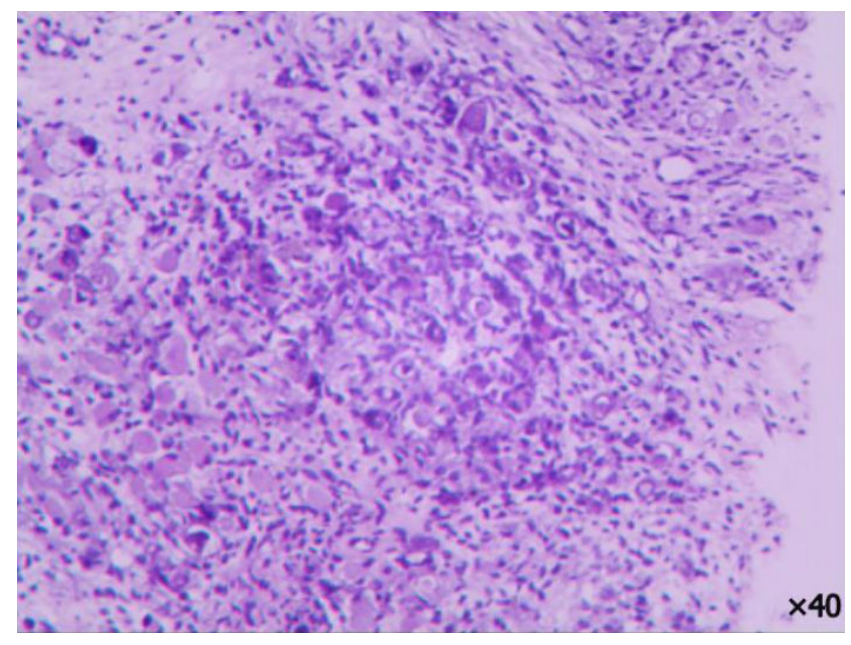

Figure 2. Ovarian tumour cytology showing several signet-ring cells (mucicarmine stain positive, $\times 40$ ).

Chemotherapy was initiated at four weeks postpartum and consisted of two cycles of oxaliplatin and tegafur on a 21-day schedule, followed by 12 cycles of oxaliplatin, tegafur, and docetaxel on a 21-day schedule. During treatment, the patient's skin became black in colour, and there was no obvious recession of her facial beard or improvement of the acne cysts. Despite all efforts, the patient died after 12 months due to intestinal obstruction and cardiac arrest.

\section{Discussion}

In China, gastric cancer has the highest incidence of all malignant tumour types in patients older than 50. While ovarian metastatic tumours can occur in female gastric cancer patients, pregnancy complicated by $\mathrm{KT}$ is extremely rare, and the prognosis is extremely poor. The 5-year survival rate is 12 to $23.4 \%$ [8] and the majority of patients die within one year [9].

The diagnosis of gastric cancer poses a challenge during pregnancy because of its extremely rare incidence, even when extremely common symptoms are presented. Nausea and vomiting are common during pregnancy, affecting $70 \%$ to $80 \%$ of all pregnant women [10]. In the case presented here, we suspected a KT after the patient was hospitalized. She appeared weak and had a facial beard, acne cysts, and a bilateral lower abdominal mass with ascites, symptoms that differ from those of primary ovarian cancer.

Although CA 125 has limited diagnostic value, it can play a role in the postoperative monitoring of patients with KT. 
Reports in the literature indicate that CA 125 levels in patients with KT are usually 33.9-150 U/ml [11]. Similarly, in our patient, the prenatal level of CA 125 was $117.50 \mathrm{U} / \mathrm{ml}$ and the postpartum level was $92.75 \mathrm{U} / \mathrm{ml}$. Pathology is the gold standard for KT diagnosis, as it allows for microscopic identification of ovarian metastases with signet ring cells and provides evidence of mucus secretion and infiltration of the ovarian stroma with sarcomatoid cells [12].

Diagnosis of KT during pregnancy is difficult, as the presenting symptoms are often attributed to the pregnancy, and further testing is invasive, with inherent risks to both maternal and foetal outcomes. In this case, the patient had presented with the clinical features prior to admission, including abdominal discomfort, stomach pain, nausea, and vomiting. However, the patient considered these to be normal pregnancyrelated symptoms and thus believed seeking medical advice was not necessary. She became alarmed and sought treatment only when she developed hematemesis and anaemia.

The patient exhibited signs of high levels of androgens that showed progression. Although these clinical observations are clearly not associated with normal pregnancy, they did not attract the attention of either the patient or her obstetrician, and the optimum time for diagnosis was missed. Although her serum testosterone level gradually decreased during the postpartum phase, the patient's masculine characteristics persisted. Ultrasonography and MRI are useful tools for characterizing adnexal masses, and may be used in conjunction with tumour markers. Timely diagnosis may improve individual outcomes [13]. There is no established optimal treatment for KT. However, results of studies using aggressive targeted chemotherapy and surgical debulking are potentially promising [14]. In late pregnancy, the survival of the foetus should be the first consideration and any treatment decisions should be made only after discussion of the advantages and disadvantages with the patient and her family. Treatment must be initiated as soon as possible in the postpartum period.

Above all, regular physical examination is very important in women of reproductive age. Obstetricians should pay special attention to persistent gastrointestinal symptoms and any other abnormal signs in patients in the mid-to-late stages of pregnancy. Such symptoms should not simply be assumed to be a result of the pregnancy itself, and careful, comprehensive ultrasound examination is necessary. Patients with obvious masculine features and an ovarian neoplasm, especially those with bilateral tumours, could have either sex cord-stromal or metastatic ovarian tumours [15].

Alternative diagnoses should also be considered in patients with worsening abdominal pain, new onset ascites, persistent hyperemesis gravidarum, or virilization. Ultrasonography and magnetic resonance imaging are useful tools for characterizing adnexal masses and may be used in conjunction with tumour marker measurements. Timely diagnosis and early detection, followed by surgery and chemotherapy during pregnancy, could result in a favourable outcome for pregnant patients with KT.
The key message in this case is the need for earlier detection and diagnosis of KT. Case studies have shown that disease detection before 24 weeks of gestation allows a wider choice of management options, including termination of the pregnancy [16]. Appropriate care should be given as soon as possible, and a full assessment should be carried out. The final decision should be made by the patient herself, her guardian, and the doctor. However, this has less impact on foetal survival, as more than $70 \%$ of pregnancies result in the delivery of a healthy baby [16].

\section{Conflicts of Interest}

The authors declare no conflict of interest.

\section{References}

1. Young R. From krukenberg to today: the ever-present problems posed by metastatic tumours in the ovary: part I. Historical perspective, general principles, mucinous tumours including the krukenberg tumour. Adv Anat Pathol 2006; 13: 205-227.

2. Singhal SR, Nanda S, Chaudhry P, Sen J, Singhal SK. Metastatic bilateral malignant ovarian tumours associated with pregnancy. Taiwan J Obstet Gynecol 2009; 48: 167-168.

3. Stojnic J, Stefanovic A, Jeremic K, Kadija S, Jeftovic M, Jeremic J. Krukenberg tumour of gastric origin in pregnancy with dismal outcome. Eur J Gynaecol Oncol 2011; 32: 356-358.

4. Al-Agha OM, Nicastri AD. An in-depth look at Krukenberg tumour: an overview. Arch Pathol Lab Med 2006; 130: 1725-1730.

5. Dueñas-García OF, Diaz-Sotomayor $\mathrm{M}$, Chanana $\mathrm{C}$. Bilateral ovarian krukenberg tumour in a full-term pregnancy. ISRN Obstet Gynecol 2011; 2011: 620380.

6. Hiremath R, Padala KP, Mahesh, Gowda G, Pailoor A. Bilateral Krukenberg Tumours Diagnosed Primarily by Transabdominal Sonography- A Case Report. J Clin Diagn Res 2015; 9: TD01-TD03.

7. Kiyokawa T, Young RH, Scully RE. Krukenberg Tumours of the ovary: a clinicopathologic analysis of 120 cases with emphasis on their variable pathologic manifestations. Am J Surg Pathol 2006; 30: 277-299.

8. Jiang R, Tang J, Cheng X, Zang RY. Surgical treatment for patients with different origins of Krukenberg tumours: outcomes and prognostic factors. Eur J Surg Oncol 2009; 35: 92-97.

9. Papantoniou N, Belitsos P, Hatzipapas I, Rodolakis A, Papaspyrou I, Antsaklis A. Excessive hirsutism in pregnancy because of Krukenberg tumour. J Matern Fetal Neonatal Med 2012; 25: 869-871.

10. Lee NM, Saha S. Nausea and vomiting of pregnancy. Gastroenterol Clin North Am 2011; 40: 309-334.

11. Luo JH, Wang R, Li YL. Krukenberg tumour secondary to gastric carcinoma with pulmonary metastasis. A case report 
and literature review. Prog Obstet Gynecol 2013; 22: 517-518.

12. Mahfoud T, Elhassani ME, Hafidi MR. Krukenberg tumour secondary to gastric carcinoma in a pregnant woman: A case report and literature review. Biol Biomed Rep 2012; 2: 32-36.

13. Co PV, Gupta A, Attar BM, Demetria M. Gastric Cancer Presenting as a Krukenberg Tumour at 22 Weeks' Gestation. J Gastric Cancer 2014; 14: 275-278.

14. Germann N, Gross-Goupil M, Wasserman E, Emile JF, Misset JL, Reynes M, Goldwasser F. The chemotherapy of metastatic gastric adenocarcinomas with hypersecretion of alpha-fetoprotein or beta-human chorionic gonadotropin: report of two cases. Ann Oncol 2002; 13: 632-636.

15. Papakonstantinou E, Liapis A, Kairi-Vassilatou E, Iavazzo C, Kleanthis CK, Kondi-Pafiti A. Virilizing ovarian
Krukenberg tumour in a 27-year-old pregnant woman. A case report and literature review. Eur J Gynaecol Oncol 2011; 32: 331-333.

16. Kim SH, Abd Halim SR, Siddiqui N, Park WH. Disseminated Cancer in Pregnancy: Krukenberg Tumour. Case Rep Obstet Gynecol 2014; 2014: 216969.

\section{*Correspondence to}

Fang-Xin Shi

Department of Obstetrics and Gynaecology

The First Affiliated Hospital of Dalian Medical University

Dalian 116011, Liaoning Province

PR China 